\title{
Operative hysteroscopy in the outpatient setting: its role within a gynaecology service
}

\author{
D. Nikolaou • G. Salman • R. Richardson
}

Received: 9 March 2008 / Accepted: 22 April 2008 /Published online: 20 May 2008

(C) Springer-Verlag 2008

\begin{abstract}
A rapid-access gynaecology service was launched at Chelsea and Westminster Hospital in January 2003, offering, among else, trans-vaginal ultrasound and outpatient hysteroscopy on a see-and-treat basis. Our aim was to review the performance of this new service in its first year. Furthermore, to critically discuss the role of such clinics within gynaecology. Prospective collection of data of the new service for the first 12 months was conducted. One hundred and one patients were seen. Hysteroscopy was attempted in 107 patients and was carried out successfully in 103 of them $(96 \%)$. In four patients, hysteroscopy was attempted but was not possible. Polyps were found in 35 patients and removed in $29(83 \%)$. The remaining six patients did not seem suitable for outpatient removal. Overall, only $10 \%$ of the patients who were seen in the clinic eventually required a general anaesthetic. Of the patients who were referred from general practitioners, $56 \%$ were discharged after assessment and treatment and $44 \%$ had follow-up appointments organised within our hospital. The outpatient hysteroscopy service is effective and potentially frees up important hospital resources, both in outpatient clinics and operating theatres.
\end{abstract}

\footnotetext{
D. Nikolaou

Assisted Conception Unit, Chelsea and Westminster Hospital, 369 Fulham Road,

London SW10 9NH, UK

G. Salman $\cdot$ R. Richardson

Chelsea and Westminster Hospital,

369 Fulham Road,

London SW10 9NH, UK

G. Salman $(\bowtie)$

94 Valiant House, Vicarage Crescent, London SW11 3LX, UK

e-mail: ghadasalman@hotmail.com
}

Keywords Outpatient hysteroscopy $\cdot$ Critical discussion

\section{Introduction}

Traditionally, the management of abnormal uterine bleeding involved dilatation and curettage under general anaesthetic. This approach had poor sensitivity for focal lesions, up to $30 \%$ of which could be missed [1]. The advent of transvaginal ultrasound (TVS) improved the diagnosis, but focal lesions could still be missed, especially in cases of two or more concomitant lesions [2]. A systematic review [3] of TVS, sonohysterography and hysteroscopy for the investigation of abnormal uterine bleeding in pre-menopausal women concluded that all three diagnostic tests were moderately accurate in detecting intrauterine pathology. The real improvement in diagnosis came from combining hysteroscopy under a general anaesthetic with directed biopsy of focal lesions [4]. Initially, this approach required a general anaesthetic and an admission to hospital, albeit for only a few hours. Progress in technology and the development of reliable, small diameter hysteroscopes and camera systems made it possible to carry out hysteroscopes without a general anaesthetic [4]. Combined with endometrial curettage or pipelle endometrial biopsy, "outpatient" hysteroscopy had similar effectiveness to hysteroscopy under general anaesthetic and curettage [5] in diagnosing generalised pathology such as endometrial hyperplasia. It was well tolerated [6] and cost-efficient [7]. Furthermore, with the development of operative hysteroscopes, directed biopsy and treatment can now be performed in the outpatient setting, resulting in excellent diagnosis of focal lesions $[8,9]$. The outpatient operative hysteroscopy service was launched at Chelsea and Westminster Hospital in January 2003, in the context of a rapid-access gynaecology 
clinic. The aim of this report was to review the performance of the new service in its first year.

\section{Materials and methods}

A prospective collection of data of the new service was carried out. Data were collected from the first clinic session on the origin of referrals, indications, findings of ultrasound and hysteroscopy, extra investigations organised and final diagnosis.

The clinic The staff involved was a consultant gynaecologist specialising in minimal access surgery, two gynaecology nurses and a trainee in minimal access surgery. In the first year, the clinic was based in our Assisted Conception Unit and used some of its facilities, including the reception desk, waiting area, the consultation room, treatment room and the recovery area. The clinic took place once a week. There were up to five appointments in each clinic session, of which the two were allocated for "rapid access" referrals, to be seen within 2 weeks. Initially, there was a 30-min time-slot per patient.

Organisation The referrals came mostly from local general practitioners (GPs) and also from other consultants within the gynaecology department, including the infertility services. After the referral was received, an appointment was generated and sent out with an information leaflet on the service. The service ran in parallel with the oncology outpatient clinic. Patients who were suspected of having gynaecological malignancies after their ultrasound and/or hysteroscopy were directed to the oncology clinic the same day.

The technique The equipment available in the treatment room included a couch with stirrups, a tower with a light source (Olympus Visera CVL-540), camera (Olympus Visera OTV-57) and printer and $30^{\circ}$ rigid hysteroscopes. We used normal saline and a pressure bag (C-Fusur 100 Medex) as a distension medium. TVS scans were performed using an Aloka SSD 1000 machine. In the treatment room, there were facilities for pulse oxymetry and electronic blood pressure and pulse monitoring, as well as oxygen supply and a resuscitation trolley. Criteria used for patient and instrument selection included parity, postmenopausal status and ability to tolerate a speculum examination. For postmenopausal or nulliparous women, typically a $1.9-\mathrm{mm}$ hysteroscope was used, whereas for pre-menopausal, parous, women, a 3-mm hysteroscope was preferred. For operative procedures, such as removal of polyp or lost intrauterine contraceptive device, usually a 3-mm hysteroscope with a
5.5- $\mathrm{mm} \mathrm{C/F}$ operative sheath with $5 \mathrm{~F}$ instrument channel was used. Most diagnostic procedures were carried out without anaesthetic, but intra-cervical block could be administered for operative procedures using Citanest 3\% with Octapressin. A Collin's speculum was used to visualise the cervix, which was then grasped with a single-toothed volsellum. The speculum was removed as soon as the hysteroscope was inserted in the cervical canal. In some cases with very narrow and atrophic vagina, the "vaginoscopic entry" was utilised [10], where the hysteroscope was introduced without a speculum or manipulation of the cervix. For the removal of polyps, rigid instruments were used down a $5 \mathrm{~F}$ operative channel. For directed biopsy of focal lesions, the "grasp" technique was used [11]: This involved removing the hysteroscope together with the forceps to maximise the amount of tissue that was sent for histology.

\section{Results}

In the first year, 121 patients were seen. The ages ranged from 23 to 83, and the mean age was 53. Sixty-four patients (53\%) were referred from GPs and 57 (47\%) were referred from other clinics. The indications for referral included: abnormal premenopausal bleeding in 24 cases (19\%), postmenopausal bleeding with or without hormone replacement therapy (HRT) in 70 cases $(58 \%)$ and sub-fertility in 27 cases $(22 \%)$. Trans-vaginal pelvic ultrasound was carried out in the clinic in 88 patients $(73 \%)$. Other patients had had an ultrasound examination carried out elsewhere or were deemed not to require one. Hysteroscopy was necessary in 107 patients and completed successfully in 103 (96\%). Intrauterine pathology was diagnosed hysteroscopically in 50 patients $(48.5 \%)$. Intrauterine polyps were found in 35 patients and removed on a see-and-treat basis in $29(83 \%)$. The remaining six were not suitable for outpatient removal, and it was not attempted. Three patients were "fast-tracked" to the oncology clinic with suspected malignancies (2.4\%). There was one endometrial malignancy, one atypia and one suspicious sample. Only 12 out of the 121 patients $(10 \%)$ eventually needed investigation or treatment under a general anaesthetic, but this included cases with resection of fibroids. A cut-off point of 4-mm endometrial thickness was used for proceeding to hysteroscopy in post-menopausal women who were not on HRT. In total, histological samples were sent for 44 patients from this clinic $(44 / 121=36 \%)$. In terms of complications, there was no case of uterine perforation and no known cases of infection or significant bleeding. There was one patient who needed atropine because of a prolonged vasovagal reaction 
$(0.9 \%)$. Of the patients who came from GPs, 36 were directly discharged back to the GP $(56 \%)$. For the rest, a treatment plan was initiated and appropriate follow-up was organised. Most patients who were referred for hysteroscopy from other gynaecology clinics had follow-up appointments with the consultants who had referred them.

\section{Discussion}

A success rate of $96 \%$ for hysteroscopy in- an outpatient setting was consistent with the literature [12]. The "see and treat" rate of over $80 \%$ for polyps was also very satisfactory. All polyps were assessed, and those that were deemed suitable for outpatient removal were all successfully removed $(100 \%)$. This achievement needs to be judged against the many difficulties the new service had to overcome in its first year: the availability and experience of nurses, availability of clinic space, the quality of ultrasound scan (USS) equipment and operators.

In a retrospective internal audit of patients who had a hysteroscopy under a general anaesthetic in our department in the same year (unpublished), each patient had five or more outpatient encounters to complete the clinical episode. This included two to three outpatient clinic appointments, a USS appointment and two appointments in relation to the hysteroscopy, one for pre-assessment and one for the procedure. On this basis, we estimate that the outpatient hysteroscopy service actually saves up to four outpatient encounters per patient: an efficiency saving for both the hospital and patient. In addition, it saves a general anaesthetic in $80 \%$ with focal intrauterine lesions.

Common practice is that the GP organises some initial investigations, then refers to an ultrasound department and a general gynaecology clinic. From this clinic onwards, referral to the outpatient hysteroscopy service is made. To make better use of its potential and increase its efficiency, the clinic needs to receive more direct referrals from GPs. It also needs to discharge more patients directly to their GP. This, however, needs to be balanced against the need of patients to receive integrated care. The risk is that the clinic could be reduced to a technician's role, only carrying out a procedure (outpatient hysteroscopy) and then requiring the GPs to manage the patient or organise onward referral. This was not our policy, as our clinic managed the patient in a "holistic" manner; hence, often, medical treatment was initiated and appropriate follow-up appointments were organised, for example in the menopause clinic.

In terms of the overall cost-effectiveness of outpatient hysteroscopy, the avoidance of a general anaesthetic and repeated hospital visits are certainly important factors: They are appropriate "savings". It is crucial however to ensure that directing the patients to the outpatient route for investigation and treatment does not mean "cutting corners" in their care. A proper outpatient hysteroscopy service needs to be adequately supported from the outset in terms of facilities, equipment, nurses and doctors. The quality of TVS, hysteroscopy and treatment, which are offered in the outpatient clinic, should match the quality of the same investigations in the "slow but sure" route of the traditional general anaesthetic service.

After review of the year's activity, it was obvious that certain types of patient were either unsuitable for a rapid access outpatient hysteroscopy service or required two or three times the allocated time to be properly managed. This included patients with complicated medical conditions or social circumstances or patients who were keen on having treatment without anaesthetic but were screened out as unsuitable for outpatient hysteroscopy. The main reason for introducing an operative outpatient hysteroscopy service is that it offers more choice for selected patients who are suitable. To further increase the efficiency of this service, "patient selection" is probably the most crucial issue. Apart from "outpatient" hysteroscopy, there is a clear need for "hysteroscopy without a general anaesthetic" in appropriately organised treatment centres, rather than the outpatient clinic, for patients who are not suitable for a general anaesthetic on medical or social grounds.

\section{Conclusions}

The operative outpatient hysteroscopy service was successfully launched in our hospital in January 2003. It has since gone from strength to strength and is now in its fourth year. It is well received and very efficient, with a $96 \%$ success rate for successfully carrying out hysteroscopy. Using rigid hysteroscopes, $80 \%$ of the total number of polyps was removed. Overall, $100 \%$ of the polyps that were assessed as suitable for outpatient removal were successfully removed in the same clinic session. At this rate of efficiency, only $10 \%$ of the total number of patients that were referred to this service eventually required a general anaesthetic, and this number included suspected malignancies and resection of fibroids. This performance should be contrasted with an ultrasound clinic or a diagnostic hysteroscopy clinic, both of which offer potentially suboptimal diagnosis without a directed biopsy and the need for onward referral for operative hysteroscopy under general anaesthetic. To maximise its efficiency potential, the operative outpatient 
hysteroscopy service needs to receive more patients directly from GPs.

\section{References}

1. Loffer FD (1989) Hysteroscopy with selective endometrial sampling compared with D \& C for abnormal uterine bleeding: The value of a negative hysteroscopic view. Obstet Gynaecol 73(1):16-20

2. Loverro G, Nappi L, Vicino M, Carriero C, Vimercati A, Selvaggi L (2001) Uterine cavity assessment in infertilie women: comparison of transvaginal sonography and hysteroscopy. Eur J Obstet Gynaecol Reprod Biol 100:67-71

3. Farquhar C, Ekeroma A, Furness S, Arroll B (2003) A systematic review of transvaginal ultrasonography, sonohysterography and hysteroscopy for the investigation of abnormal uterine bleeding in premenopausal women. Acta Obstet Gynecol Scand 82:493-504

4. Valle R (1999) Office hysteroscopy. Clin Obstet Gynecol 42 (2):276-289

5. Tahir MM, Bigrigg MA, Browning JJ, Brookes ST, Smith PA (1999) A randomised controlled trial comparing transvaginal ultrasound, outpatient hysteroscopy and endometrial biopsy with inpatient hysteroscopy and curettage. $\mathrm{Br} \mathrm{J}$ Obstet Gynaecol 106:1259-1264
6. Kremer C, Duffy S, Moroney M (2000) Patient satisfaction with outpatient hysteroscopy versus day case hysteroscopy: randomised controlled trial. BMJ 320:279-282

7. Marsh F, Kremer C, Duffy S (2004) Delivering an effective outpatient service in gynaecology. A randomised controlled trial analysing the cost of outpatient versus daycase hysteroscopy. BJOG 111:243-248

8. Bettocchi S, Ceci O, Pansini MV, Pellegrino A, Marello F, Nappi L (2002) Advanced operative office hysteroscopy without anaesthesia: analysis of 501 cases treated with a 5 Fr. Bipolar electrode. Hum Reprod 17(9):2435-2438

9. Bettocchi S, Nappi L, Ceci O, Selvaggi L (2003) What does "diagnostic hysteroscopy" mean today? The role of the new techniques. Curr Opin Obstet Gynecol 15:303-308

10. Sharma M, Taylor A, di Spiezio Sard A, Buck L, Mastrogamvrakis G, Kosmas I, Tsirkas P, Magos A (2005) Outpatient hysteroscopy: traditional versus the "no-touch" technique. BJOG 112 (7):963-967

11. Bettochi S, Di Venere R, Pansini N, Pansini MV, Pellegrino A, Santamato S, Ceci O (2002) Endometrial biopsies using smalldiameter hysteroscopes and $5 \mathrm{~F}$ instruments: how can we obtain enough material for a correct histologic diagnosis. J Am Ass Gynecol Laparosc 9:290-292

12. Lotfallah H, Farag K, Hassan I, Watson R (2005) One-stop hysteroscopy clinic for postmenopausal bleeding. J Reprod Med 50(2):101-107 